\title{
Microarray-based analysis of the gene expression profile in GC-1 spg cells transfected with spermatogenesis associated gene 12
}

\author{
YITING LIN, ZHIWEN LIU, XUANMING LIU, YUNSHENG ZHANG, ZHUOXIAN RONG and DAN LI \\ Department of Life Science, School of Biology, Hunan University, Changsha 410082, P.R. China
}

Received September 24, 2012; Accepted November 16, 2012

DOI: $10.3892 /$ ijmm.2012.1225

\begin{abstract}
The unique differentiation mechanisms of spermatogenesis suggest the existence of cell type- and stagespecific molecules. Herein, a microarray-based approach was used to identify changes in the gene expression profile in mouse GC-1 spg germ cells transfected with spermatogenesis associated gene 12 (SPATA12). One hundred and eighty-two upregulated genes and 104 downregulated genes with fold changes of $\geq 2$ or $\leq 0.5(\mathrm{P} \leq 0.05)$ in expression were identified. Ten genes were selected for validation of the microarray results using quantitative RT-PCR. The real-time quantitative RT-PCR results were consistent with that of the microarray. The gene ontology (GO) terms for the biological functions of the differentially expressed genes induced by SPATA12 included binding activity and immune response. Biological pathway analysis identified several related pathways which are associated with immune responses, cell adhesion and the developmental process. In addition, we observed that SPATA12 may interact with the $\beta$-catenin signaling pathway and that SPATA1 2 may negatively regulate $\beta$-catenin signaling during spermatogenesis.
\end{abstract}

\section{Introduction}

Infertility affects approximately $15 \%$ of couples worldwide, and the male factor is at least partly responsible in approximately $50 \%$ of infertile couples (1). Genetic abnormalities, i.e. obstacles to spermatogenesis, are thought to account for $15-30 \%$ of male factor infertility (2). Spermatogenesis is the process by which male germ cells undergo a complex differentiation where morphological alterations lead to the formation of differentiated sperm. The unique differentiation mechanisms of spermatogenesis suggest the existence of cell

Correspondence to: Dr Dan Li, Department of Life Science, School of Biology, Hunan University, Changsha 410082, P.R. China E-mail: lidanie@hotmail.com

Key words: spermatogenesis associated gene 12, microarray, expression profile, $\beta$-catenin, GC-1 spg cells type- and stage-specific molecules. With the advent of genetics and molecular biology, a variety of genes involved in spermatogenesis have been identified during the last decade (3-9).

Recently, we identified a novel protein referred to as spermatogenesis associated gene 12 (SPATA12) $(10,11)$. In the testis, SPATA12 is specifically expressed in spermatocytes, spermatids and spermatozoa, and may be involved in the development of testicular maturation. In contrast, the SPATA12 gene is located on chromosome $3 \mathrm{p} 14$. Chromosomal abnormalities including homozygous deletions, loss of heterozygosity and expressional deficiencies in genes located at 3p14 have been frequently reported in many tumor types, suggesting that this locus is likely to contain tumour-suppressor genes $(12,13)$. SPATA12 was also found to be absent in testicular germ cell tumors such as seminoma, yolk sac, teratoma and embryonal carcinoma. Flow cytometric analysis of SPATA12 in both mouse GC-1 spg germ cells and human HeLa cells indicates that the expression of the SPATA12 gene may delay G1 to $\mathrm{S}$ phase progression in the cell cycle. In addition, SPATA12 was shown to inhibit tumor cell colony formation. These findings suggest that SPATA12 could be an inhibitor suppressing cell proliferation in the process of germ cell development and in tumorigenesis. However, the transcriptional regulations of SPATA12 in spermatogenesis remain unclear.

The emerging technology of cDNA microarray hybridization offers the possibility of providing a rapid, high-throughput method for the efficient and accurate simultaneous expression measurement of thousands of genes (14-17). Studies based on microarray or expressed sequence tag analyses have been successfully used during spermatogenesis, and have aided in the understanding of the molecular mechanisms and genetic determinants of male infertility (18-20). Based on this background, we hypothesized that analysis of the gene expression profile induced by SPATA12 in GC-1 spg germ cells may contribute to an understanding of the function of SPATA12 and the possible pathways in which SPATA12 is involved during spermatogenesis.

\section{Materials and methods}

Cell line. The mouse GC-1 spg germ cell line (ATCC CRL-2053) was cultured in Dulbecco's modified Eagle's medium supplemented with $10 \% \mathrm{FBS}, 100 \mu \mathrm{g} / \mathrm{ml}$ penicillin- 
Table I. Primers used for real-time RT-PCR and RT-PCR experiments.

\begin{tabular}{|c|c|c|c|}
\hline Gene name & Forward primer $\left(5^{\prime} \rightarrow 3^{\prime}\right)$ & Reverse primer $\left(5^{\prime} \rightarrow 3^{\prime}\right)$ & $\begin{array}{l}\text { Product size } \\
\text { (bp) }\end{array}$ \\
\hline Gapdh1 & GAAGGGTGGAGCCAAAAGG & TTGCTGACAATCTTGAGTGAGTTG & 111 \\
\hline $\mathrm{Ccl5}$ & GCCCACGTCAAGGAGTATTTCT & TCTCTGGGTTGGCACACACTT & 100 \\
\hline Fbxo39 & AGCCTGAGGAGTTGCTATTTCAGT & ACGTTAACTTCTGCAGGGTGTTC & 100 \\
\hline Wnt10a & CTTCAGCCGAGGTTTTCGAGA & CCGCAAGCCTTCAGTTTACC & 108 \\
\hline Rtp4 & CATCTTTGGGTGAGAAGGTGACT & GAGATCTGGGTGGTTTTACTTTGTG & 120 \\
\hline Sp100 & AGCTACAACCACAGTCCCCT & TCCTGTCCTTTTCCGTCTTCTAA & 111 \\
\hline Zbp1 & GACGGACAGACGTGGAAGATC & TTGACCGGATTGTGCTGACA & 110 \\
\hline Gys1 & CGCTGGAAGGGTGAGCTTT & GAAGTGGGCAACCACATACG & 156 \\
\hline Mtss1 & ATGGAGGCTGTGATCGAGAAG & TCCGGCTTTGTTTATGAAGTCTT & 114 \\
\hline Selenbp1 & AGCCAGGTCATCCACAGGTT & ACTTCGTGCTGTCCCCAAAG & 100 \\
\hline Ndrg1 & CACACAACATTTTGCTGTCTGC & GCCAACTGATCCATTGAGGGG & 100 \\
\hline Cyclin B1 & TGGCCTCACAAAGCACATGA & GCTGTGCCAGCGTGCTAATC & 77 \\
\hline Cyclin D1 & TAGGCCCTCAGCCTCACTC & CCACCCCTGGGATAAAGCAC & 80 \\
\hline Cyclin E1 & AATTGGGGCAATAGAGAAGAGGT & TGGAGCTTATAGACTTCGCACA & 161 \\
\hline$\beta$-catenin & GGCAACCCTGAGGAAGAAGA & CACTGGTGACCCAAGCATTTT & 597 \\
\hline Gapdh2 & TTCAACGGCACAGTCAAGG & TGAAGTCGCAGGAGACAACC & 694 \\
\hline SPATA12 & CGCGGATCCATGTCCAGTTCTGCTCTGACT & CCCAAGCTTGCAGGATTATTATTGATTACAG & 607 \\
\hline
\end{tabular}

The primers of Gapdh1 were used for quantitative RT-PCR, while those for Gapdh2 were used for RT-PCR.

streptomycin, and was maintained in $5 \% \mathrm{CO}_{2}$ and a $95 \%$ humidified air atmosphere at $37^{\circ} \mathrm{C}$.

Transient transfection. Transfections were performed with Lipofectamine 2000 (Invitrogen). Cells were plated to 50-70\% confluent culture in each well of a 6 -well plate or a $60-\mathrm{mm}$ dish $24 \mathrm{~h}$ before transfection. According to the manufacturer's instructions, the GC-1 spg cells were transfected with $4 \mu \mathrm{g}$ pRevTRE plasmid or pRevTRE-SPATA12 plasmid, respectively.

RNA isolation. Total RNA was extracted with the TRIzol reagent (Invitrogen) according to the manufacturer's protocol, digested by RNase-free DNase (Fermentas), dissolved in diethyl pyrocarbonate-treated water, and stored at $-80^{\circ} \mathrm{C}$ prior to use. For quality control, RNA purity and integrity were evaluated by agrose gel electrophoresis and the OD260/OD280 ratio.

Microarray assay and data analysis. 32K Mouse Genome Array (CapitolBio Corporation, Beijing, China), covering 99\% of the current assembly of the mouse genome and including 32,256 Oligo DNA with 70 mer length, was applied to investigate the possible changes in the mRNA level in GC-1 spg cells following SPATA12 gene transfection by comparison to control GC-1 spg cells. Total RNA was extracted using TRIzol reagent (Invitrogen) and then applied to synthesize Cy3- or Cy5-conjugated dUTP-labeled cDNA probe using the RNA Fluorescence Labeling Core kit (M-MLV version 2.0; Takara, Dalian, China) and following the manufacturer's instructions. Hybridization, scanning, and data extraction were conducted at CapitalBio Corporation. The number of genes affected by SPATA12 was determined using the scatter plots of control GC-1 spg cells vs. GC-1 spg cells transfected with SPATA12 (named GC-1 spg-SPATA12 cells). In addition, identified genes were also categorized specifically using biological process ontology terms and Kyoto Encyclopedia of Genes and Genomes (KEGG) biological pathways using the software Molecule Annotation System 2.0 (http//:bioinfo.capitalbio.com/MAS/) (CapitolBio Corporation).

RT-PCR and SYBR-Green real-time PCR analysis. For cDNA synthesis, $2 \mu \mathrm{g}$ of total RNA was reverse transcribed using M-MLV reverse transcriptase (Promega). Then PCR was performed in a $10 \mu \mathrm{l}$ reaction volume containing $5.7 \mu \mathrm{l}$ of nuclease-free water, $0.1 \mu \mathrm{l}$ of Takara Taq $(5 \mathrm{U} / \mu \mathrm{l}), 1 \mu \mathrm{l}$ of 10X PCR buffer, $0.8 \mu \mathrm{l}$ of dNTP mixture $(2.5 \mathrm{mM}), 2 \mu \mathrm{l}$ of cDNA and $0.2 \mu \mathrm{l}$ each of the $20 \mu \mathrm{M}$ gene-specific primers. After initial denaturation for $10 \mathrm{~min}$ at $94^{\circ} \mathrm{C}, 27-30$ cycles of PCR were performed. Each cycle consisted of a denaturing period $\left(30 \mathrm{sec}\right.$ at $\left.94^{\circ} \mathrm{C}\right)$, an annealing phase $\left(55-60^{\circ} \mathrm{C}\right)$, and an extension period $\left(60 \mathrm{sec}\right.$ at $\left.72^{\circ} \mathrm{C}\right)$. After the last cycle, all samples were incubated for an additional $10 \mathrm{~min}$ at $72^{\circ} \mathrm{C}$. PCR products were separated by $1.5 \%$ agarose gel electrophoresis, and the DNA bands were stained with ethidium bromide. RT-PCR signals were normalized to the signals of the murine glyceraldehyde-3-phosphate dehydrogenase (gapdh) gene.

For quantitative RT-PCR, a SYBR-Green real-time RT-PCR protocol (Invitrogen) was applied using an MX3000 (Stratagene, USA) instrument. PCR was performed in a $10 \mu \mathrm{l}$ reaction volume containing $2.2 \mu \mathrm{l}$ of nuclease-free water, $5 \mu \mathrm{l}$ of Mix, $2 \mu \mathrm{l}$ of cDNA and $0.4 \mu \mathrm{l}$ each of the $2.5 \mu \mathrm{M}$ genespecific primers. The PCR profile was $95^{\circ} \mathrm{C}$ for $5 \mathrm{~min}$ followed by $94^{\circ} \mathrm{C}$ for $30 \mathrm{sec}, 58^{\circ} \mathrm{C}$ for $20 \mathrm{sec}$, and $72^{\circ} \mathrm{C}$ for $20 \mathrm{sec}$ for 40 cycles, with a final extension at $72^{\circ} \mathrm{C}$ for $10 \mathrm{~min}$ and storage at $4^{\circ} \mathrm{C}$. The level of gene mRNA was evaluated in automated analysis by MxPro. All the primers for RT-PCR are shown in Table I. 


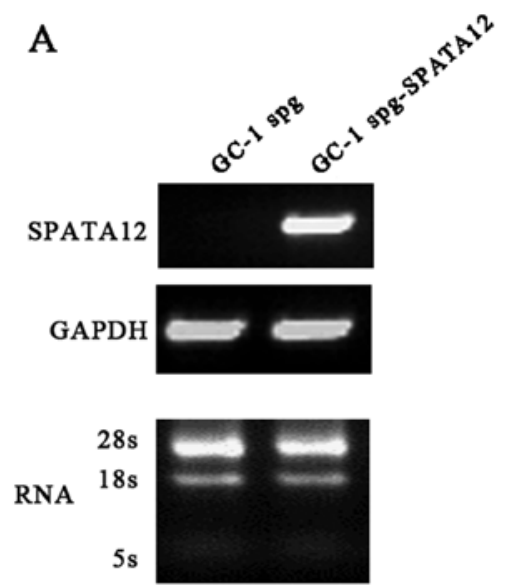

B

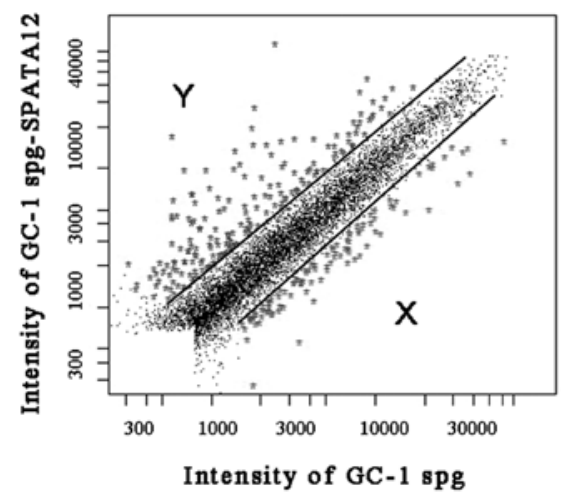

Figure 1. Global gene expression induced by SPATA12 in GC-1 spg cells. (A) The RT-PCR analysis of SPATA12 mRNA in GC-1 spg and GC-1 spg-SPATA12 cells . The quality of RNA used for the microarray analysis was assessed by agarose gel electrophoresis. (B) The log-log scatter-plot of the microarray results. Plots in region Y represent upregulated genes, while plots in region X represent downregulated genes in GC-1 spg following SPATA12 transfection.

Table II. Selected genes with differential expression between GC-1 spg and GC-1 spg-SPATA12 cells.

\begin{tabular}{|c|c|c|c|c|}
\hline Term description & Symbol & Ratio & Gene name & Gene function \\
\hline \multirow[t]{6}{*}{ Upregulated genes } & Ccl5 & 32.0033 & $\begin{array}{l}\text { Chemokine (C-C motif) } \\
\text { ligand } 5\end{array}$ & $\begin{array}{l}\text { Cytokine activity; chemokine activity; } \\
\text { chemoattractant activity; immune regulation }\end{array}$ \\
\hline & Rtp4 & 11.7258 & Receptor transporter protein 4 & Unknown \\
\hline & Zbp1 & 8.7427 & Z-DNA binding protein 1 & $\begin{array}{l}\text { Left-handed Z-DNA binding; RNA binding; } \\
\text { double-stranded RNA adenosine deaminase } \\
\text { activity; innate immune response }\end{array}$ \\
\hline & Fbxo39 & 6.7244 & F-box protein 39 & Cancer/testis antigen \\
\hline & Sp100 & 4.1610 & SP100 nuclear antigen & Tumorigenesis; immunity; gene regulation \\
\hline & Wnt10a & 2.1549 & $\begin{array}{l}\text { Wingless related MMTV } \\
\text { integration site } 10 \mathrm{a}\end{array}$ & $\begin{array}{l}\text { Signal transducer activity; receptor binding; } \\
\text { embryogenesis; carcinogenesis }\end{array}$ \\
\hline \multirow[t]{4}{*}{ Downregulated genes } & Gys1 & 0.4613 & Glycogen synthase 1 (muscle) & $\begin{array}{l}\text { Catalytic activity; glycogen (starch) synthase } \\
\text { activity; protein binding; transferase activity; } \\
\text { transferase activity, transferring glycosyl groups }\end{array}$ \\
\hline & Mtss1 & 0.3599 & Metastasis suppressor 1 & Unknown \\
\hline & Ndrg 1 & 0.2371 & $\begin{array}{l}\mathrm{N} \text {-myc downstream } \\
\text { regulated gene } 1\end{array}$ & $\begin{array}{l}\text { Stress responses; hormone responses; } \\
\text { cell growth; cell differentiation; p53-mediated } \\
\text { caspase activation and apoptosis }\end{array}$ \\
\hline & Selenbp1 & 0.1536 & Selenium binding protein 1 & $\begin{array}{l}\text { Selenium binding; selenium-dependent role in } \\
\text { ubiquitination/deubiquitination-mediated } \\
\text { protein degradation }\end{array}$ \\
\hline
\end{tabular}

Statistical analysis. Statistical analysis was carried out using the Student's t-test. P-values $\leq 0.05$ were considered to indicate statistically significant results.

\section{Results}

Global gene expression induced by SPATA12 in GC-1 spg cells. Global gene expression levels induced by SPATA12 in GC-1 spg cells from the microarray analysis are represented in Fig. 1. The differentially expressed genes with fold changes of $\geq 2$ or $\leq 0.5(\mathrm{P} \leq 0.05)$ were analyzed using t-test and $\mathrm{P}$-value and clustered with the software package Cluster 3.0. Our data showed that the expression of 286 out of 32,256 genes was altered after SPATA12 was expressed. Of these, 182 genes were upregulated and 104 genes were downregulated specifically.

Verification of the differentially expressed genes from the microarray data. To test the reliability of the microarray data, the expression patterns of 10 selected genes (including 6 upregulated genes and 4 downregulated genes) (Table II) 

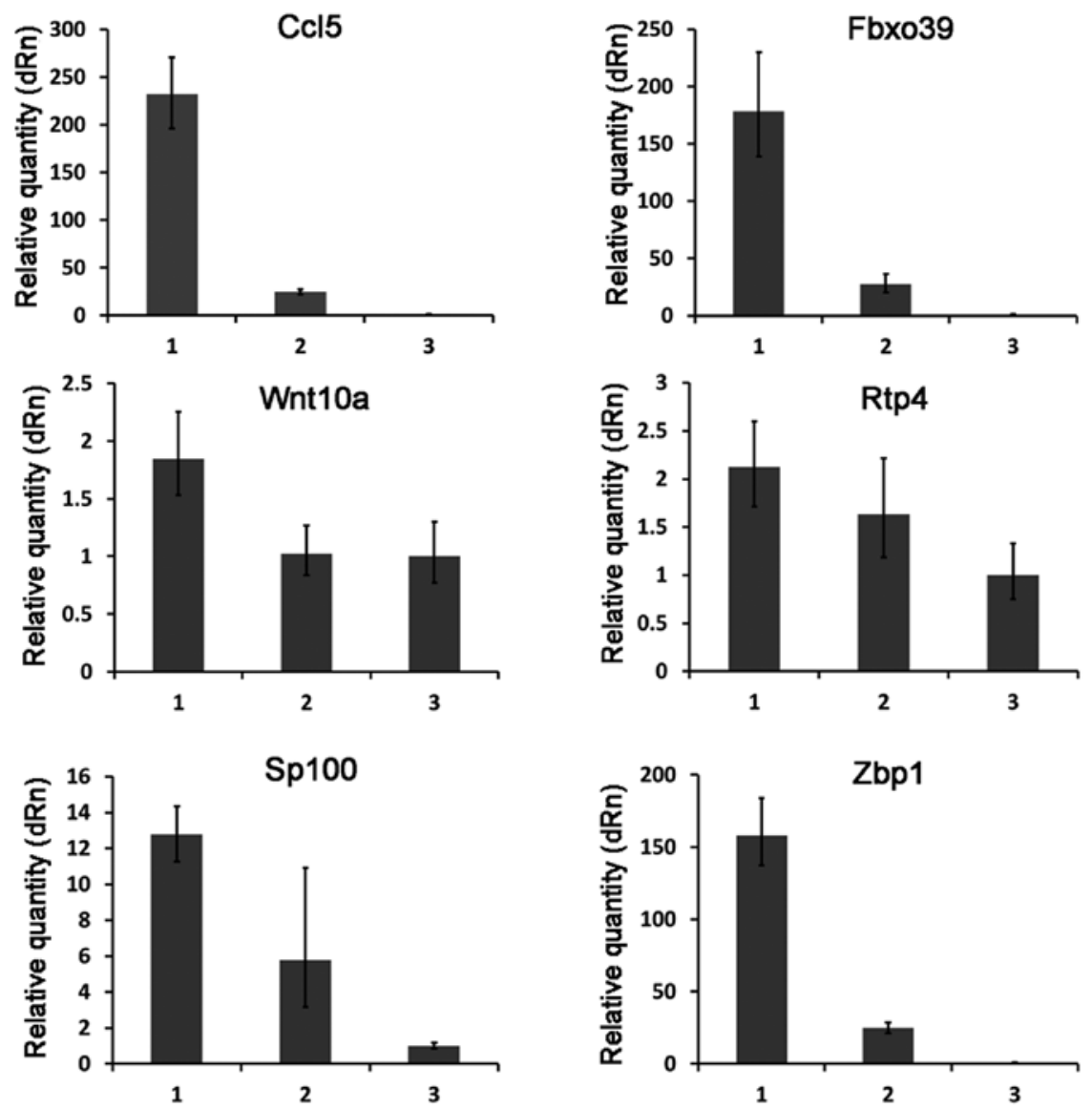

Figure 2. Validation of the expression of upregulated genes by real-time RT-PCR. Histogram: bar 1, GC-1 spg cells transfected with the pRevTRE-SPATA12 plasmid; bar 2, GC-1 spg cells transfected with the pRevTRE plasmid; bar 3, control GC-1 spg cells.
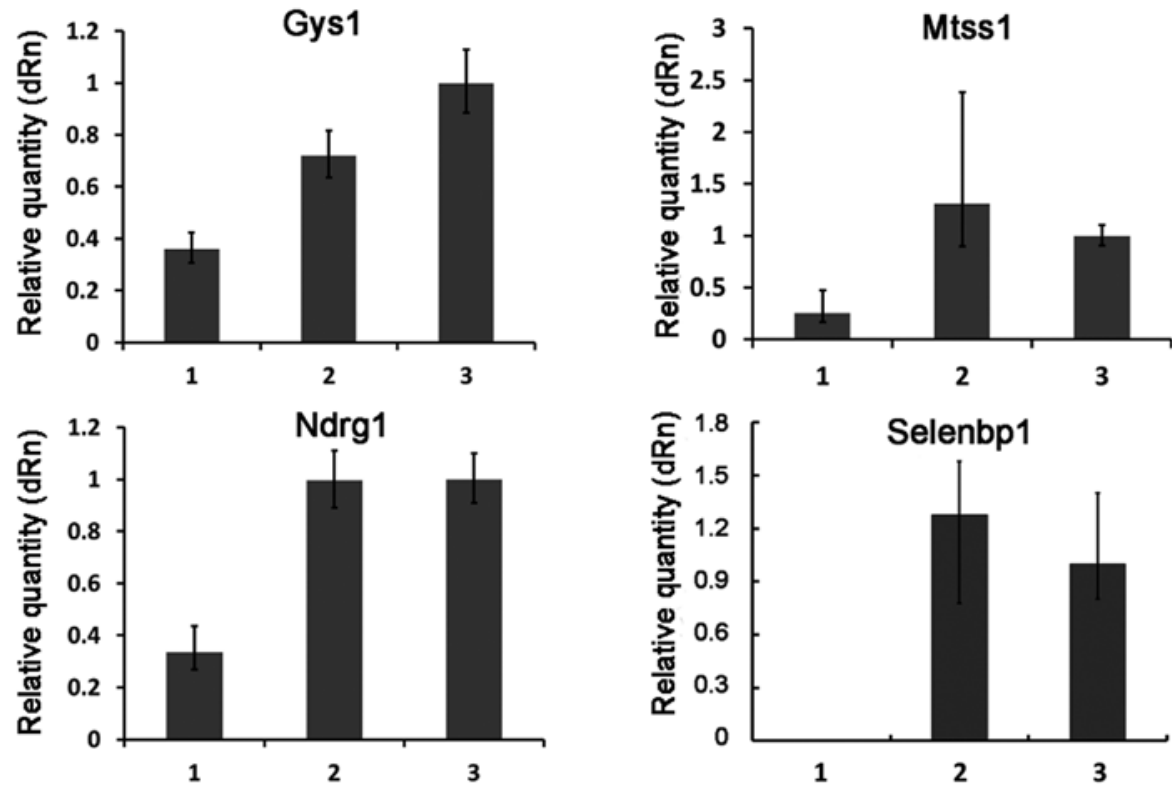

Figure 3. Validation of the expression of the downregulated genes by real-time RT-PCR. Histogram: bar 1, GC-1 spg cells transfected with the pRevTRESPATA12 plasmid; bar 2, GC-1 spg cells transfected with the pRevTRE plasmid; bar 3, control GC-1 spg cells.

were examined by quantitative real-time RT-PCR. The overall profile of gene expression by real-time RT-PCR analysis was similar to that revealed by the microarray data for all the selected genes (Figs. 2 and 3).
Gene ontology $(G O)$ analysis of the microarray data. To gain insight into the potential functional consequences of the SPATA12-induced expression in GC-1 spg cells, GO analysis was applied to distribute genes into groups according to 
A

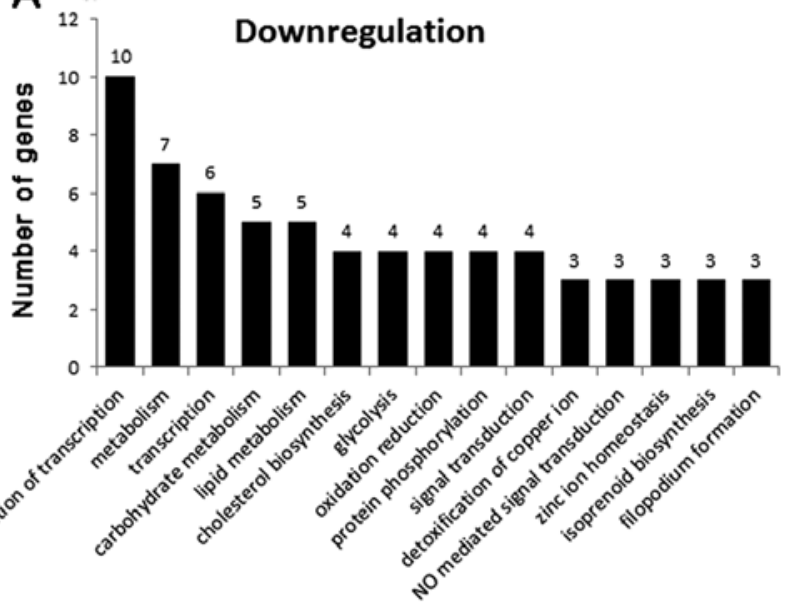

C

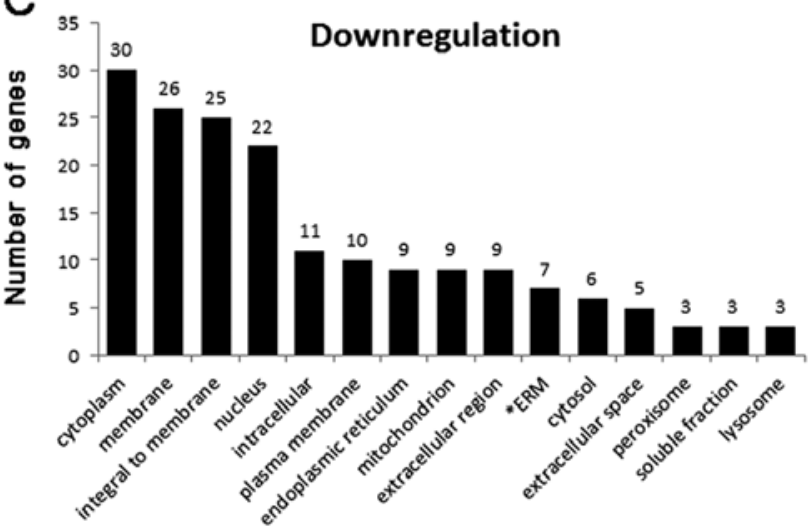

E

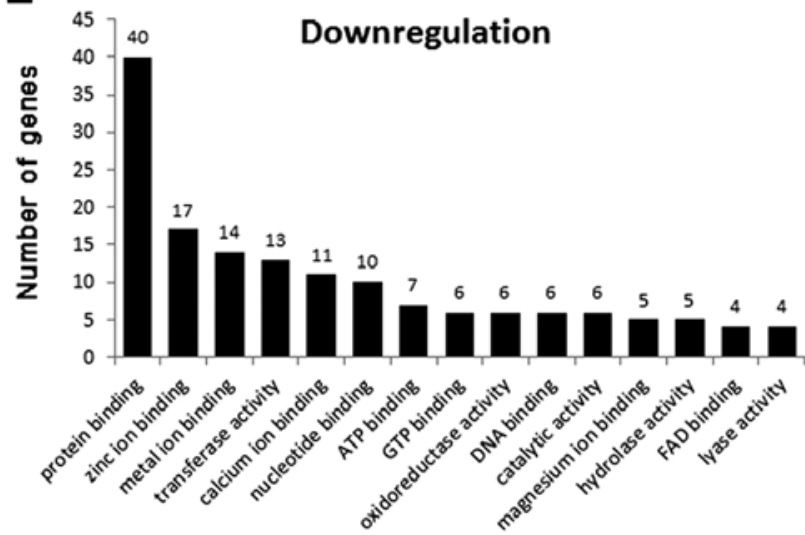

B
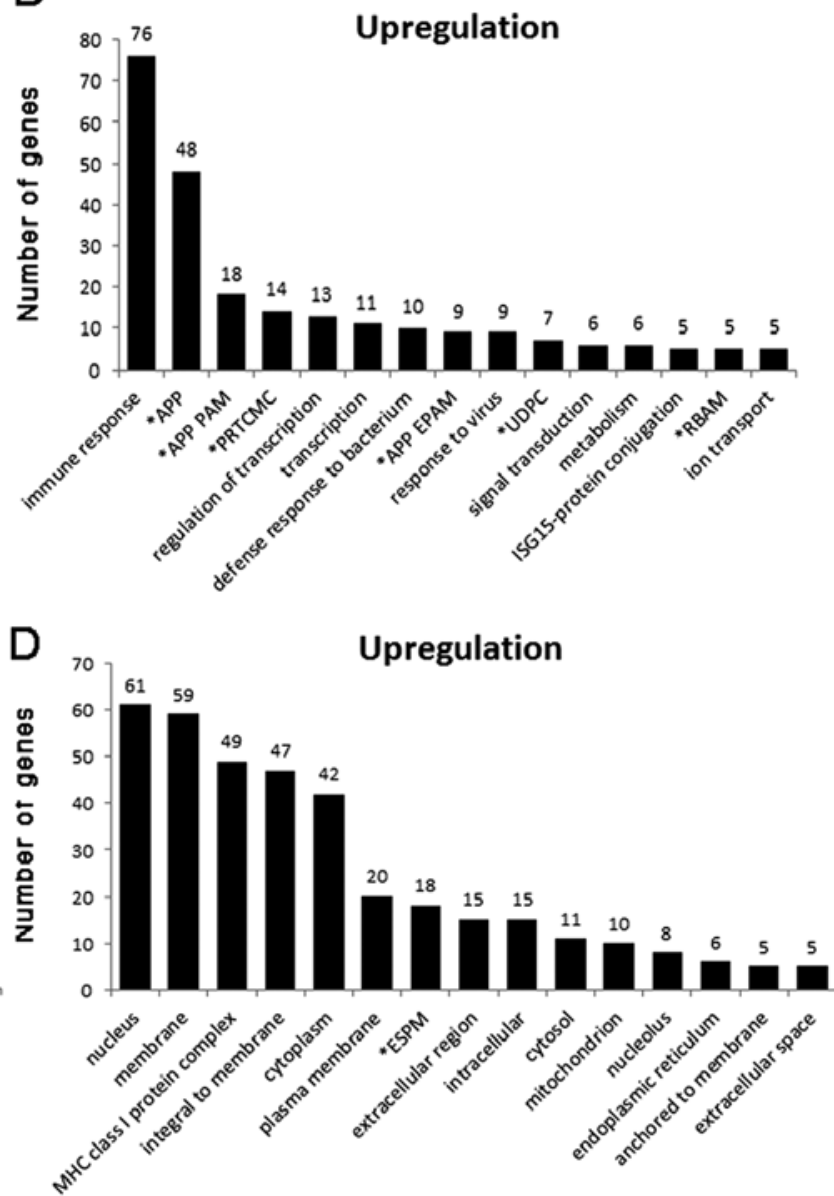

F

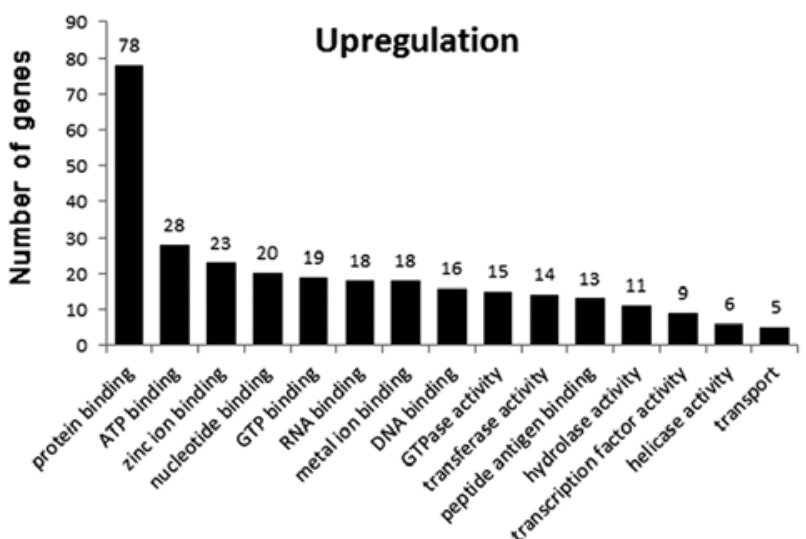

Figure 4. The number of SPATA12-induced genes in each of the (A and B) biological process, $(\mathrm{C}$ and D) cellular component and (E and F) molecular functional categories described in the Gene Ontology website. (A, C and E) Downregulated genes; (B, D and F) upregulated genes. "APP, antigen processing and presentation; "APP PAM, antigen processing and presentation of peptide antigen via MHC class I; "PRTCMC, positive regulation of T cell mediated cytotoxicity; "APP EPAM, antigen processing and presentation of exogenous peptide antigen via MHC class I; "UDPC, ubiquitin-dependent protein catabolism; "RBAM, response to bacterium associated molecule; "ERM, endoplasmic reticulum membrane; ${ }^{*}$ ESPM, external side of plasma membrane.

biological process, molecular function and cellular component, respectively.

Functional analysis using $\mathrm{GO}$ of the biological process group revealed that genes related to immune response, antigen processing and presentation, positive regulation of $\mathrm{T}$ cellmediated cytotoxicity, transcription regulation, and defense response to bacterium were upregulated in the SPATA12transfected GC-1 spg cells (Fig. 4A and B). This suggests that based on the GO terms these upregulated genes combined with SPATA12 may play important roles in the physiology of the immune response. In contrast, genes related to carbohydrate metabolism, lipid metabolism, cholesterol biosynthesis and glycolysis were downregulated in the SPATA12-transfected cells, which indicates that based on these GO terms these genes affected by SPATA12 may be involved in metabolic processes. 
Table III. Selected pathways related with SPATA12 identified by biological pathway analysis.

\begin{tabular}{|c|c|c|}
\hline Pathway name & Total & Gene (ratio) \\
\hline Antigen processing and presentation & 12 & $\begin{array}{l}\text { H2-Q10 (2.36), H2-Q7 (2.79), H2-T9 (2.49), H2-T3 (2.01), } \\
\text { H2-Q6 (2.02), Tap1 (2.3442), Psme2 (2.4576), H2-K1 (2.28), } \\
\text { H2-Q1 (2.92), Psme2b-ps (2.4576), B2m (2.5449), H2-T23 (2.24) }\end{array}$ \\
\hline Cell adhesion molecules (CAMs) & 9 & $\begin{array}{l}\text { H2-Q10 (2.36), H2-Q7 (2.79), H2-T9 (2.49), H2-T3 (2.01), } \\
\text { H2-Q6 (2.02), Itgb7 (2.73), H2-K1 (2.28), H2-Q1 (2.92), H2-T23 (2.24) }\end{array}$ \\
\hline Type I diabetes mellitus & 8 & $\begin{array}{l}\mathrm{H} 2-\mathrm{T} 23 \text { (2.24), H2-Q1 (2.92), H2-K1 (2.28), H2-Q6 (2.02), H2-T3 (2.01), } \\
\mathrm{H} 2-\mathrm{T} 9 \text { (2.49), H2-Q7 (2.79), H2-Q10 (2.36) }\end{array}$ \\
\hline Toll-like receptor signaling pathway & 5 & $\begin{array}{l}\text { Myd88 (2.0637), Cxcl10 (5.5093), Stat1 (5.04), } \\
\text { Cd14 (2.0947), Cc15 (32.0033) }\end{array}$ \\
\hline MAPK signaling pathway & 4 & Ddit3 (2.0538), Cd14 (2.0947), Dusp6 (2.9646), Map4k2 (0.4768) \\
\hline Biosynthesis of steroids & 3 & Sqle (0.4314), Pmvk (0.4707), Fdft1 (0.4985) \\
\hline ECM-receptor interaction & 3 & Col6a1 (0.4919), Col5a3 (0.4825), Itgb7 (2.7347) \\
\hline Jak-STAT signaling pathway & 3 & Isgf3g (3.57), Stat1 (5.04), Stat2 (2.55) \\
\hline Cytokine-cytokine receptor interaction & 3 & Cc15 (32.0033), Vegfa (0.4651), Cxc110 (5.5093) \\
\hline Natural killer cell mediated cytotoxicity & 2 & H2-T23 (2.24), H2-K1 (2.28) \\
\hline $\mathrm{T}$ cell receptor signaling pathway & 1 & Pdk1 (0.4713) \\
\hline Wnt signaling pathway & 1 & Wnt10a (2.15) \\
\hline
\end{tabular}
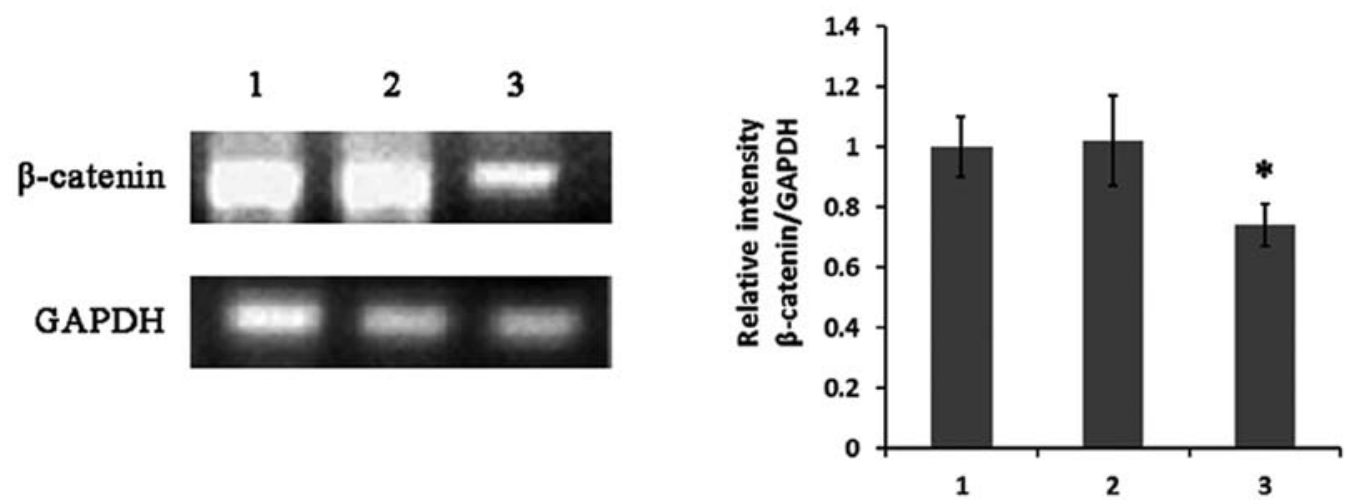

Figure 5. Expression of $\beta$-catenin in GC-1 spg cells transfected with SPATA12. Lane 1, control GC-1 spg cells; lane 2, GC-1 spg cells transfected with the pRevTRE plasmid; lane 3, GC-1 spg cells transfected with the pRevTRE-SPATA12 plasmid. Data are means \pm SD ( $n=4$ ). ${ }^{*} \mathrm{P}<0.05$ vs. control (group 1) by t-test.

In the cellular component group (Fig. 4C and D), GO analysis showed that the major differentially expressed genes, including 61 upregulated and 22 downregulated genes, were located in the nucleus. These data indicate that SPATA12 may play roles in the nucleus through interacting with genes associated in these GO categories. This observation was consistent with our previous bioinformatics analysis report, which predicted that SPATA12 probably functions as a testis-specific nuclear protein involved in spermatogenesis (21).

We also noted that these genes (molecular function group) (Fig. 4E and F), either upregulated or downregulated in SPATA12-transfected cells, were mostly involved in protein binding, ATP/GTP binding, zinc ion binding or DNA/RNA binding, indicating that the function of SPATA12 may be related with the activity of 'binding'.
Pathway analysis and the expression change in $\beta$-catenin signaling induced by SPATA12. The analysis of the differential expression of genes led us to wonder whether these genes may represent related pathways in which SPATA12 is involved. Thus, biological pathway analysis was applied to characterize the unique gene networks associated with SPATA12 in germ cells. Most genes associated with immune-related pathways such as antigen processing and presentation, cell adhesion, $\mathrm{T}$ cell receptor signaling pathway, and development-related pathways such as MAPK, Jak-STAT and Wnt, were significantly overexpressed which provides evidence that both immune responses and developmental processes may be associated with various functions of SPATA12 (Table III). Changes in the expression of Wnt signaling-related genes such as $\beta$-catenin supported this possibility. Semi-quantitative RT-PCR results showed that the 

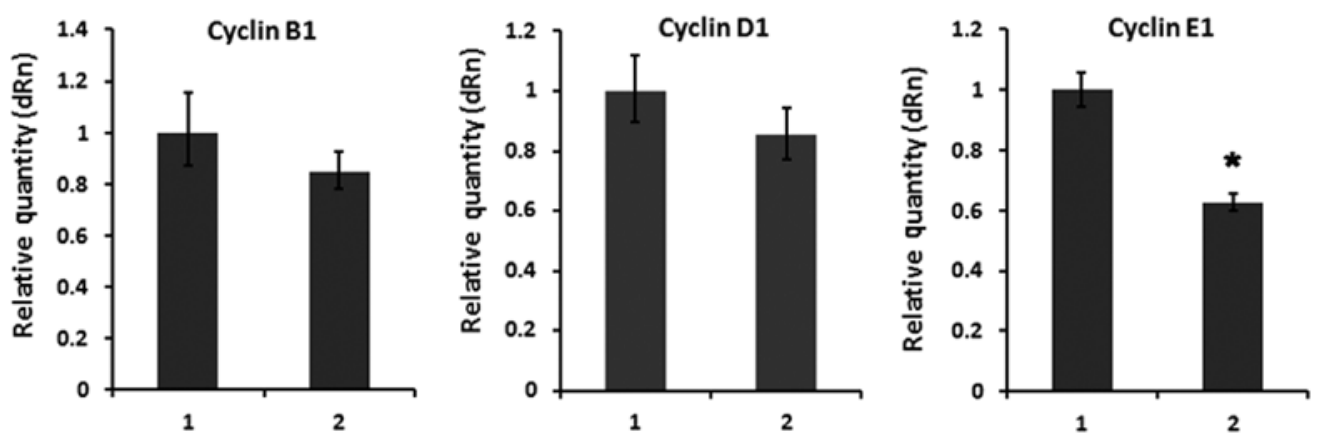

Figure 6. Expression of cell cycle-related genes in the GC-1 spg cells transfected with SPATA12. Histogram: bar 1, GC-1 spg cells transfected with the pRevTRE plasmid; bar 2, GC-1 spg cells transfected with the pRevTRE-SPATA12 plasmid. Data are means $\pm \mathrm{SD}(\mathrm{n}=4)$. ${ }^{*} \mathrm{P}<0.05$ vs. control (group 1$)$ by $\mathrm{t}$-test.

expression of $\beta$-catenin was obviously downregulated (Fig. 5) and its downstream target cell cycle gene cyclin El was strongly decreased (Fig. 6) in GC-1 spg cells transfected with SPATA12.

\section{Discussion}

High-density cDNA microarrays provide an important tool to study the global patterns of gene expression. For example, it has been used to understand interactions in a given cell, tissue and organism under normal and diseased states $(22,23)$, or it has been used for gene discovery and function $(24,25)$. Spermatogenesis is a complex process of cell development and differentiation that requires the highly regulated expression of multiple genes (26). Characterization and functional analysis of new testis-specific genes related to spermatogenesis is of momentous physiological and pathological significance in order to understand the molecular mechanisms of spermatogenesis. Herein, cDNA microarray was used to identify the upregulated or downregulated genes affected by SPATA12 and obtain a global overview on the expression patterns of these genes, aimed at acquiring a further understanding of the function of SPATA12 and the possible pathways in which SPATA12 is involved.

We identified 182 upregulated genes and 104 downregulated genes with a fold change of $\geq 2$ or $\leq 0.5$ ( $\mathrm{P} \leq 0.05$ ) in expression. Through quantitative real-time RT-PCR, we confirmed the expression of 10 (Ccl5, Fbxo39, Wnt10a, Rtp4, Sp100, Zbp1, Gys1, Mtss1, Selenbp1 and Ndrg1) of the differentially expressed genes. These genes along with their GO categories identified as being differentially expressed following induction by SPATA12 may be of significant biological interest.

Gene ontology studies provide biologically meaningful information regarding genes including the cellular location, molecular function and biological process. Fig. 4 lists each GO category and the specific number of genes in their respective category that were differentially expressed following induction by SPATA12. Biological pathway analysis identified several related pathways such as antigen processing and presentation, cell adhesion, MAPK, Jak-STAT and Wnt. The differential expression of these signaling pathway-related genes may also show that SPATA12 is associated with immune responses in germ cell development.

Alterations in the expression of $\mathrm{Wnt} / \beta$-catenin signalingrelated genes support this possibility. Wnt/ $\beta$-catenin signaling is one of the most important developmental signaling pathways that control cell fate decisions and tissue patterning during early and late embryonic development. $\beta$-catenin is a key component of the Wnt/ $\beta$-catenin signaling pathway (27-30) and was previously reported to be expressed in the plasma membrane and cytoplasm of germ cells during testis development. Suppression of Wnt/ $\beta$-catenin signaling is necessary for the normal development of primordial germ cells since stabilization of $\beta$-catenin in germ cells was found to delay cell cycle progression resulting in germ cell deficiency (31). Here, we showed that expression of SPATA12 by transfection downregulated $\beta$-catenin in GC- 1 spg cells. Studies in the literature reported that the reduction in $\beta$-catenin level was accompanied by inhibition of its transactivation potential and downregulation of downstream target genes, such as $M y c$, cyclin D1 and cyclin E1 $(28,32,33)$. In the present study, our data suggest that SPATA12 negatively regulates cell cyclerelated gene cyclin E1, which indicates that SPATA12 inhibits cell proliferation via downregulation of $\beta$-catenin in GC-1 spg cells. These results were shown to be of particular relevance between SPATA12 and the $\beta$-catenin signaling pathway.

Taken together, the present study demonstrated alterations in the gene expression profile of GC-1 spg cells transfected with SPATA12. The functional classification of these genes and their expression profiles provide useful information to understand the transcriptional regulation of SPATA12. A number of genes, GO categories and biological pathways such as immune responses may be associated with the function of SPATA12. Moreover, our study showed that SPATA12 may interact with the $\beta$-catenin signaling pathway and SPATA12 could negatively regulate $\beta$-catenin signaling during spermatogenesis.

\section{Acknowledgements}

This study was supported by the National Natural Science Foundation of China (nos. 30872763, 81270735) and the Fundamental Research Funds for the Central Universities of China (no. 531107040314).

\section{References}

1. O'Flynn O'Brien KL, Varghese AC and Agarwal A: The genetic causes of male factor infertility: a review. Fertil Steril 93: 1-12, 2010 . 
2. Ferlin A, Raicu F, Gatta V, Zuccarello D, Palka G and Foresta C: Male infertility: role of genetic background. Reprod Biomed Online 14: 734-745, 2007

3. Coussens M, Maresh JG, Yanagimachi R, Maeda G and Allsopp R: Sirtl deficiency attenuates spermatogenesis and germ cell function. PLoS One 3: e1571, 2008.

4. Takeuchi A, Mishina Y, Miyaishi O, Kojima E, Hasegawa T and Isobe K: Heterozygosity with respect to Zfp148 causes complete loss of fetal germ cells during mouse embryogenesis. Nat Genet 33: 172-176, 2003.

5. Zhou CX, Zhang YL, Xiao L, et al: An epididymis-specific betadefensin is important for the initiation of sperm maturation. Nat Cell Biol 6: 458-464, 2004.

6. Roy A, Yan W, Burns KH and Matzuk MM: Tektin3 encodes an evolutionarily conserved putative testicular microtubules-related protein expressed preferentially in male germ cells. Mol Reprod Dev 67: 295-302, 2004.

7. Takahashi T, Tanaka H, Iguchi N, et al: Rosbin: a novel homeoboxlike protein gene expressed exclusively in round spermatids. Biol Reprod 70: 1485-1492, 2004

8. Kissel H, Georgescu MM, Larisch S, Manova K, Hunnicutt GR and Steller H: The Sept4 septin locus is required for sperm terminal differentiation in mice. Dev Cell 8: 353-364, 2005.

9. Zhu H, Zhu JX, Lo PS, et al: Rescue of defective pancreatic secretion in cystic-fibrosis cells by suppression of a novel isoform of phospholipase C. Lancet 362: 2059-2065, 2003.

10. Dan L, Lifang Y and Guangxiu L: Expression and possible functions of a novel gene SPATA12 in human testis. J Androl 28 502-512, 2007.

11. Liu ZW, Lin YT, Liu XM, Yu WW, Zhang YS and Li D Experimental study of inhibition of tumor cell proliferation by a novel gene SPATA12. Zhong Nan Da Xue Xue Bao Yi Xue Ban 37: 222-227, 2012 (In Chinese).

12. Ji L, Minna JD and Roth JA: 3 p21.3 tumor suppressor cluster: prospects for translational applications. Future Oncol 1: 79-92, 2005.

13. Riquelme E, Tang M, Baez S, Diaz A, Pruyas M, Wistuba II and Corvalan A: Frequent epigenetic inactivation of chromosome $3 \mathrm{p}$ candidate tumor suppressor genes in gallbladder carcinoma. Cancer Lett 250: 100-106, 2007.

14. Kim HJ, Joo HJ, Kim YH, et al: Systemic analysis of heat shock response induced by heat shock and a proteasome inhibitor MG132. PLoS One 6: e20252, 2011.

15. Korkor MT, Meng FB, Xing SY, Zhang MC, Guo JR, Zhu XX and Yang P: Microarray analysis of differential gene expression profile in peripheral blood cells of patients with human essential hypertension. Int J Med Sci 8: 168-179, 2011.

16. Roy Choudhury D, Small C, Wang Y, Mueller PR, Rebel VI, Griswold MD and McCarrey JR: Microarray-based analysis of cell-cycle gene expression during spermatogenesis in the mouse. Biol Reprod 83: 663-675, 2010.
17. King HC and Sinha AA: Gene expression profile analysis by DNA microarrays: promise and pitfalls. JAMA 286: 2280-2288, 2001.

18. Zeng S and Gong Z: Expressed sequence tag analysis of expression profiles of zebrafish testis and ovary. Gene 294: 45-53, 2002

19. Xiao P, Tang A, Yu Z, Gui Y and Cai Z: Gene expression profile of 2058 spermatogenesis-related genes in mice. Biol Pharm Bull 31: 201-206, 2008

20. Lo LJ, Zhang ZH, Hong N, Peng JR and Hong YH: 3640 unique EST clusters from the medaka testis and their potential use for identifying conserved testicular gene expression in fish and mammals. PLoS One 3: e3915, 2008.

21. LI D and LU GX: Identification and expression of a novel human testis-specific gene by digital differential display. Chin Med J 117: 1791-1796, 2004.

22. Sridhar K, Ross DT, Tibshirani R, Butte AJ and Greenberg PL: Relationship of differential gene expression profiles in CD $34^{+}$ myelodysplastic syndrome marrow cells to disease subtype and progression. Blood 114: 4847-4858, 2009.

23. Creekmore AL, Silkworth WT, Cimini D, Jensen RV, Roberts PC and Schmelz EM: Changes in gene expression and cellular architecture in an ovarian cancer progression model. PLoS One 6: e17676, 2011.

24. Mattison J, Weyden L, Hubbard T and Adams DJ: Cancer gene discovery in mouse and man. Biochim Biophys Acta 1796 140-161, 2009.

25. Chen F, Zhu HH, Zhou LF, et al: Genes related to the very early stage of ConA-induced fulminant hepatitis: a gene-chip-based study in a mouse model. BMC Genomics 11: 240, 2010.

26. Eddy EM: Male germ cell gene expression. Recent Prog Horm Res 57: 103-128, 2002.

27. Davidson G: The cell cycle and Wnt. Cell Cycle 9: 1667-1668, 2010.

28. Chang H, Gao F, Guillou F, Taketo MM, Huff V and Behringer RR: Wt1 negatively regulates $\beta$-catenin signaling during testis development. Development 135: 1875-1885, 2008.

29. Olmeda D, Castel S, Vilaró S and Cano A: Beta-catenin regulation during the cell cycle: implications in $\mathrm{G} 2 / \mathrm{M}$ and apoptosis. Mol Biol Cell 14: 2844-2860, 2003.

30. Peifer $M$ and Polakis P: Wnt signalling in oncogenesis and embryogenesis: a look outside the nucleus. Science 287: 16061609,2000

31. Kimura T, Nakamura T, Murayama K, et al: The stabilization of beta-catenin leads to impaired primordial germ cell development via aberrant cell cycle progression. Dev Biol 300: 545-553, 2006

32. Tetsu $\mathrm{O}$ and McCormick F: Beta-catenin regulates expression of cyclin D1 in colon carcinoma cells. Nature 398: 422-426, 1999.

33. Botrugno OA, Fayard E, Annicotte JS, et al: Synergy between LRH-1 and beta-catenin induces G1 cyclin-mediated cell proliferation. Mol Cell 15: 499-509, 2004 